\title{
ON AUTONOMOUS CONTROL SYSTEMS ON CERTAIN MANIFOLDS ${ }^{1}$
}

\author{
CHAO-CHU LIANG
}

\begin{abstract}
Let $M^{n}$ be a compact $C^{\infty}$ manifold, $n>4$, admitting a vector field with every orbit a circle. Then there exists a completely controllable set $S$ consisting of two nonsingular $C^{\infty}$ vectors $X$ and $Y$ such that every orbit of $X$ is a circle.
\end{abstract}

An autonomous control system on a smooth manifold $M$ is the same as a set of vector fields on $M$. A set $S$ of vector fields on a smooth manifold $M$ is said to be controllable if for every pair $\left(m, m^{\prime}\right)$ of points of $M$ there exists a trajectory of $S$ from $m$ to $m^{\prime}$. Here a trajectory of $S$ is a curve which is an integral curve (orbit) of some $X \in S$ or a finite concatenation of such curves such that a trajectory of $S$ run in reverse is not allowed. (We refer the readers to [2] for details.)

In [2], N. Levitt and H. J. Sussmann showed that on every connected paracompact manifold of class $C^{k}, 2 \leqslant k \leqslant \infty$, or $k=\omega$, there exists a completely controllable set $S$ consisting of two vector fields of class $C^{k-1}$.

For simplicity, we assume that all the manifolds, vector fields, etc., considered here are of class $C^{\infty}$.

A manifold $M$ is called closed if it is compact and without boundary. Let $D^{k}$ denote the $k$-dimensional disk and $S^{k-1}$ its boundary.

The purpose of this paper is to prove the following theorem:

THEOREM. If a connected closed n-dimensional manifold $M^{n}, n>4$, admits a vector field $X_{0}$ with every orbit a (nondegenerate) circle, then there exists a completely controllable set $S$ consisting of two nonsingular vectors $X$ and $Y$ such that every orbit of $X$ is a circle and $Y$ has finitely many closed orbits.

We first give a brief sketch of the proof.

According to [1], $M$ can be decomposed as a union of round handles $R_{k}=S^{1} \times$ $D^{k} \times D^{n-k-1}$. Each round $k$-handle $R_{k}$ is supplied with a vector field

$$
V=d / d t-\sum x_{i} \partial / \partial x_{i}+\sum y_{j} \partial / \partial y_{j}
$$

where $(t, x, y) \in S^{1} \times D^{k} \times D^{n-k-1}$. A point $p \in R_{k}$ can be moved along a trajectory of $V$ arbitrarily close to the closed orbit $S^{1} \times 0 \times 0$ if and only if $p \in S^{1} \times D^{k} \times 0$. Modifying the vector field $V$ on each $R_{k}$, we will get a

Received by the editors February 20, 1978.

AMS (MOS) subject classifications (1970). Primary 49E15.

Key words and phrases. Completely controllable set of vector fields, orbits, trajectory, round handle decomposition of a manifold.

${ }^{1}$ Supported by the University of Kansas General Research Fund.

(c) 1980 American Mathematical Society $0002-9939 / 80 / 0000-0212 / \$ 02.00$ 
nonsingular vector field $W$ such that for $k>0$ (respectively $k=0$ ) any trajectory of $W$ approaching $S^{1} \times 0 \times 0$ (respectively any trajectory of $\left.W\right)$ meets $S^{1} \times\left(x_{1}\right.$ axis) (respectively $S^{1} \times\left(y_{1}\right.$-axis)) in $R_{k}$. By patching up the vector fields $W$ on the $R_{k}$ 's as in [1], we construct a nonsingular vector field $Y$ on $M$ with finitely many closed orbits $\left\{C_{i}\right\}$, where $C_{i}$ corresponds to $S^{1} \times 0 \times 0$ on each $R_{k}$. By using the standard transversality argument, we may assume that near $C_{i}$ for each $t \in S^{1}$ the $x_{1}$-axis (or $y_{1}$-axis) forms part of an orbit of $X$. Then we construct a sequence of diffeomorphisms $f_{1}, \ldots, f_{N-1}$ of $M$ to itself such that the $C_{i}$ 's are connected by the orbits of the vector field $X=f_{(N-1)^{*}} \ldots f_{1^{*}}\left(X_{0}\right)$. The set $\{X, Y\}$ is showed to be completely controllable.

Let $V$ denote the vector

$$
d / d t-\sum_{i=1}^{k} x_{i} \partial / \partial x_{i}+\sum_{j=1}^{n-k-1} y_{j} \partial / \partial y_{j}
$$

on $R_{k}=S^{1} \times D^{k} \times D^{n-k-1}$, where the $x_{i}$ 's and $y_{j}$ 's denote the standard coordinate functions on $R^{k}$ and $R^{n-k-1}$ respectively.

LEMMA 1. For $k>0$, there exists a vector field $W$ on $R_{k}$ such that $W$ has $S^{1}=S^{1} \times 0 \times 0$ as its only closed orbit, and $S^{1} \times\left(x_{1}\right.$-axis -0$)$ is reachable from every trajectory except $S^{1}$.

Proof. Assume that $k>1$, let $B_{j} \subseteq D^{k}$ be the 2-dimensional disk spanned by $x_{1}$-axis and $x_{j}$-axis, $1<j \leqslant k$. We write $r B_{j}$ for the concentric disk of radius $r$. For small $\theta_{0}>0$, we may construct a diffeomorphism $g_{\theta_{j}}: B_{j} \rightarrow B_{j}$ for each $0<\theta<\theta_{0}$ such that $g_{\theta_{j}}$ fixes the complement of $\frac{3}{4} B_{j}, g_{\theta_{j}} \mid \frac{1}{4} B_{j}=$ the rotation by a degree of $\theta$, and $G_{j}\left(\theta, x_{1}, x_{j}\right)=g_{\theta_{j}}\left(x_{1}, x_{j}\right), 0<\theta<\theta_{0}$, is an isotopy with $g_{0}=$ identity. Then we define $g_{\theta_{j}}$ for arbitrary $\theta>0$ by $g_{\theta_{j}}=g_{r, j} g_{\theta_{0, j}}^{p}$, where $\theta=p \theta_{0}+r$ with $p$ an integer and $r>0$. The diffeomorphism $g_{\theta j}$ induces a diffeomorphism $h_{\theta j}$ on $D^{k} \times$ $D^{n-k-1}$ by fixing the remaining coordinates. We also write

$$
H_{j}\left(\theta, x_{1}, \ldots, x_{k}, y_{1}, \ldots, y_{n-k-1}\right)=h_{\theta j}\left(x_{1}, \ldots, y_{n-k-1}\right) .
$$

We construct an isotopy

$$
F_{j}:[4 j \pi, 4(j+1) \pi] \times D^{k} \times D^{n-k-1} \rightarrow D^{k} \times D^{n-k-1}
$$

as follows:

$$
F_{j}(t, x, y)= \begin{cases}H_{j}(t-4 j \pi, x, y) & \text { for } t \in[4 j \pi,(4 j+1) \pi], \\ H_{j}(\pi, x, y) & \text { for } t \in[(4 j+1) \pi,(4 j+2) \pi] \\ H_{j}((4 j+3) \pi-t, x, y) & \text { for } t \in[(4 j+2) \pi,(4 j+3) \pi] \\ H_{j}(0, x, y) & \text { for } t \in[(4 j+3) \pi,(4 j+4) \pi]\end{cases}
$$

The map $F_{j}$ induces a diffeomorphism $K_{j}$ from $[4 j \pi, 4(j+1) \pi] \times D^{k} \times D^{n-k-1}$ to itself, where $K_{j}(t, x, y)=\left(t, F_{j}(t, x, y)\right)$. By gluing together $K_{j}, 2<j<k$, on the 
common boundaries, we obtain a diffeomorphism $K$ of $[8 \pi, 4 n \pi] \times D^{k} \times D^{n-k-1}$ to itself. Identifying $8 \pi$ with $4 n \pi$, we thus have a diffeomorphism $\bar{K}$ of $S^{1} \times D^{k}$ $\times D^{n-k-1}$ (geometrically, $\bar{K}$ is given by twisting $\frac{1}{4} B_{j}$ along $S^{1}$ by $180^{\circ}$, and then twisting it back $180^{\circ}$ successively for $\left.2 \leqslant j \leqslant n-1\right)$. We define $W$ to be $\bar{K}_{*}(V)$ on $R_{k}$. Q.E.D.

The same proof yields the following lemma:

LEMMA 2. There exists a nonsingular vector field $W$ on $R_{0}=S^{1} \times D^{0} \times D^{n-1}=$ $S^{1} \times D^{n-1}$ with every trajectory except $S^{1}$ leaving $R_{0}$, and every trajectory meets $S^{1} \times\left(y_{1}\right.$-axis $)$.

For a round $k$-handle $R_{k}=S^{1} \times D^{k} \times D^{n-k-1}$, we write $\partial_{-} R_{k}=S^{1} \times S^{k-1}$ $\times D^{n-k-1}$, and $\partial_{+} R_{k}=S^{1} \times D^{k} \times S^{n-k-2}[1$, p. 42].

Proof of THE TheOrem. Since $M$ supports a nonsingular vector field $X_{0}$, its Euler number vanishes. According to [1], for $n \geqslant 4, M$ admits a round handle decomposition, that is, $M$ can be written as $R_{0}^{1}+\cdots+R_{0}^{b_{1}}+\cdots+R_{n-1}^{1}$ $+\cdots+R_{n-1}^{b_{n-1}}$, where each $R_{k}^{i}$ denote a round $k$-handle attached to the boundary of the stuff on the left to it, successively (using $\partial_{-} R_{k}^{i}$ as the attaching region at each stage), [1, p. 43]. Near $\partial_{-} R_{k}^{i}$, when $k>0, W$ points inwards (into $R_{k}^{i}$ ). Hence we may use the argument in [1, pp. 52-53] to patch up the $W$ 's to construct a vector field $Y$ on $M$ with finitely many closed orbits $\left\{C_{k}^{j}\right\}$, corresponding to the core $S^{1}=S^{1} \times 0 \times 0$ in $R_{k}^{j}$. Furthermore, by the standard transversality argument, we may assume that the orbits of $X_{0}$ meet each $C_{k}^{j}$ transversely. Therefore, near each $C_{k}^{j}=S^{1}$, for each $t \in S^{1}$ the $x_{1}$-axis (or $y_{1}$-axis when $k=0$ ) forms part of an orbit of $X_{0}$.

Recall that $M=R_{0}^{1}+\cdots+R_{0}^{b_{1}}+\cdots+R_{n-1}^{b_{n-1}}$ with $C_{k}^{j} \subseteq R_{k}^{j}$. We order the $C_{k}^{j}$,s from left to right in this decomposition, and denote them by $\left\{C_{j}\right\}_{j=0}^{N}$.

Now we are going to construct a sequence of diffeomorphisms $f_{j}, 0 \leqslant j \leqslant N-1$, from $M$ to itself such that $C_{j}$ and $C_{j+1}$ are connected by an orbit $\gamma_{j}$ of $f_{j *}\left(X_{j}\right)=$ $X_{j+1}$, and $f_{j}\left(\gamma_{i}\right)=\gamma_{i}$ when $i<j$. Let $\beta_{j}$ be an orbit of $X_{j}$ meeting $C_{j}$, and $p$ a point on $\beta_{j}$ but not on $C_{j}$ (such a point exists, because of the transversality). We embed a curve $\partial:[0,1] \rightarrow M$ with $\partial(0)=p$ and $\partial(1)=p^{\prime}$, a point on $C_{j+1}$. Since $n \geqslant 4$, we may assume that $\partial((0,1))$ does not intersect any of the other $C_{i}$ 's and $\gamma_{i}$ 's with $i<j$. Let $U_{j}$ be a tubular neighborhood of $\partial([0,1])$ in $M$ with $U_{j} \cap C_{j+1}=l$, a line segment, and $U_{j}$ is disjoint from all the other $C_{t}$ 's and $\gamma_{i}$ 's with $i<j$. As in [1, pp. 44-45], we construct an isotopy $F_{j}$ with support in $U_{j}$ from the identity to a diffeomorphism $f_{j}$ with $f_{j}(p)=p^{\prime}$ (geometricaly, we drag $p$ to $p^{\prime}$ along the path $\partial$ ). The orbit $\gamma_{j}=f_{j}\left(\beta_{j}\right)$ of the vector field $f_{j^{*}}\left(X_{j}\right)=X_{j+1}$ connects $C_{j}$ and $C_{j+1}$. We apply this argument successively to get a vector field $X_{N}$ with an orbit $\gamma_{j}$ connecting $C_{j}$ and $C_{j+1}$ for each $j$ with $0<j<N-1$.

By using the transversality argument again if necessary, we perturb $X_{N}$ near each $C_{k}^{j}$ to get a vector field $X$ (also with every orbit a circle) such that near $C_{k}^{j}$ for each $t \in S^{1}$ the $x_{1}$-axis (or $y_{1}$-axis when $k=0$ ) forms parts of an orbit of $S$.

We claim that $\{X, Y\}$ forms a completely controllable system. Given any two points $m, m^{\prime}$ on $M$. From the description of $Y$, we see that $m^{\prime}$ must be on a 
trajectory coming out from some $R_{0}^{i}$. On the other hand, $m$ is on a trajectory approaching some $C_{k}^{j} \subseteq R_{k}^{j}$ with $k>0$. We can reach $C_{r}^{i}$ from $C_{k}^{j}$ by a sequence of the trajectories $\gamma_{j}$ of $X$ constructed above and some closed orbits of $Y$. From Lemma 1, Lemma 2, and the last paragraph, we see that $C_{k}^{j}$ is reachable from $m$, and $m^{\prime}$ is reachable from $C_{0}^{i}$. Hence $m^{\prime}$ is reachable from $m$ by trajectories (in the positive direction) of the system $\{X, Y\}$. Q.E.D.

Any closed connected manifold $M^{n}, n>4$, which is the total space of an $S^{1}$-bundle satisfies the conclusion of the theorem. For example, the odd-dimensional sphere $S^{2 k+1}$ is the total space of the Hopf bundle $S^{1} \rightarrow S^{2 k+1} \rightarrow \operatorname{CP}(k)$, where $\mathrm{CP}(k)$ denotes the $k$-dimensional complex projective space.

For $n=3$, the proof shows that if $M^{3}$ satisfies the additional condition that it admits a round handle decomposition, then the conclusion also holds. For example, $S^{3}$ can be written as $S^{1} \times D^{2}+D^{2} \times S^{1}$, one round 0 -handle and one round 2-handle.

\section{REFERENCES}

1. D. Asimov, Round handles and non-singular Morse-Smale flows, Ann. of Math. (2) 102 (1975), 41-54.

2. N. Levitt and H. J. Sussmann, On controllability by means of two vector fields, SIAM J. Conrol 13 (1975), 1271-1281.

Department of Mathematics, University of Kansas, LaWrence, Kansas 66045 